\title{
Development of a Hybridized Diversity Combiner over Nakagami Fading Channel
}

\author{
Zachaeus K. Adeyemo \\ Electronic and Electrical Engineering Department \\ Ladoke Akintola University of Technology \\ Ogbomoso, Nigeria. \\ Email: zkadeyemo@lautech.edu.ng \\ Samson I. Ojo \\ Electronic and Electrical Engineering Department \\ Ladoke Akintola University of Technology \\ Ogbomoso, Nigeria. \\ Simeon B. Ebinaiye \\ Electronic and Electrical Engineering Department \\ Ladoke Akintola University of Technology \\ Ogbomoso, Nigeria. \\ Olasunkanmi F. Oseni \\ Electronic and Electrical Engineering Department \\ Ladoke Akintola University of Technology \\ Ogbomoso, Nigeria.
}

Received: 27 January 2019; Accepted: 20 February 2019; Published: 08 May 2019

\begin{abstract}
Wireless communication system is of paramount importance in the world of telecommunication infrastructure and is expected to be a leading role in the development of a nation. However, the system is characterized by multipath propagation effects that lead to variability of the received signal thereby degrading the performance. Equal Gain Combiner (EGC) being used to address this problem is associated with hardware complexity that result in long processing time, while Threshold Combiner (TC) with low processing time has poor performance. Hence, in this paper, a hybridized Diversity Combiner (DC) consisting of EGC and TC, (TC-EGC) with a closed form expression over Nakagami fading channel is developed. TC-EGC is derived using the conventional EGC and TC at the receiver. Randomly generated bits used as source data are modulated using M-ary Quadrature Amplitude Modulation (M-QAM) and transmitted over Nakagami channel after filtering. The faded signals generated at varying paths ' $L$ ' $(2,3,4)$ are scanned by TC to select the strongest paths. The outputs from the three TCs are combined by EGC to obtain the received signal which is converted to baseband through demodulation. A mathematical expression using the Probability Density Function (PDF) of Nakagami fading channel at varying paths ' $L$ ' for Outage Probability (OP) is also derived. The technique is simulated using Matrix Laboratory (version 7.2). The performance is evaluated using Signal-to-Noise Ratio (SNR), Outage Probability (OP) and Processing Time (PT). The study shows that the
\end{abstract}

TC-EGC gives lower OP and PT values when compared with conventional EGC and TC, with reduction in hardware complexity. The TC-EGC developed can be used to enhance the performance of wireless communication system.

Index Terms - Equal Gain Combining (EGC), Threshold Combining (TC), Hybridized TC-EGC, Outage Probability (OP), Processing Time (PT)

\section{INTRODUCTION}

Communication is a process by which people express their thoughts, feelings or exchange information or messages. It is as old as the creation of human being. The various means of communication in the olden days are namely: Man to man communication which involves special movement of man from one place to another, secondly, movement of message carries within the same town and from one location to another. The third one is the coded communication which was popular among the traditional rulers and secret cult's members in the olden day. It involves a carrier who might not have known the content of messages [15]. Frantic efforts have been made to improve the various means of communication among people. Nowadays, effort is being made to improve the means of communication over the barriers encountered due to advances in technology. Modern communication 
Involves the use of wired and space as media of transmission between the sender and receiver. Due to the fact that wireless communication is not limited by distance, it has been receiving much attention in the field of technology. The system used to process the message or information consists of a transmitter and receiver. The performance of wireless communication systems is mainly governed by the wireless environment. As opposed to the typically static and predictable characteristics of a wired channel, the wireless channel is unpredictable and this makes an exact analysis of the wireless communication system often difficult. In wireless communication, the information to be transmitted through space is referred to as radio waves. The radio waves are mainly affected by various mechanisms such as reflection, diffraction, scattering and so on $[15,18,24]$.

Nowadays, wireless communications are undergoing a huge expansion due to their immense applications in various economic sectors of every nation. The systems to achieve this are subject to severe multipath propagation which is a phenomenon that occurs when a transmitted signal propagates over different paths due to obstructions along the path in terrestrial environment and upper atmosphere [2]. Multipath fading results in fluctuation of the received signal and this fluctuation could be so severed as to produce a signal which is below the sensitivity of the receiver, causing poor reception of the signal and degrades the performance. Thus, compensation is required to mitigate the effect of this multipath propagation [7]. A lot of compensation techniques have been in existence to mitigate the effect of fading due to multipath propagation. Some of these are space diversity, equalization of various forms, empirical prediction models and so on. Space diversity is of different forms, among which is diversity combining. Diversity Combining Technique (DCT) is a well-known approach to mitigate the effect of fading in wireless channels. This is popularly used to combine the independent fading paths to reduce the effects of fading $[4,26]$. It improves the receiver performance by processing a number of statistically independent copies of the same information over fading channel. DCT is a classical and powerful technique that skillfully combines multiple replicas of the received signal to combat multipath impairments [13].

There are several combining techniques such as Maximal Ratio Combining (MRC), Equal Gain Combining (EGC), Threshold Combining (TC) and Selection Combining (SC) $[7,14,15]$. The results of past research on diversity combining technique revealed that, though EGC provides better performance than other combining techniques but at the expense of hardware complexity. Though, Threshold Combining (TC) and Selection Combining (SC) have low hardware complexity with poor performance when compared to MRC and EGC. The complexity of EGC receivers depends on the number of diversity paths combined, which can be quite high, especially for multipath diversity of wideband Code Division Multiple Access (CDMA) signals [7]. Threshold Combining (TC) uses only one path out of the $L$ available paths and hence, does not fully exploit the amount of diversity offered by the channel. Bridging the gap between these two techniques is of importance in addressing the fading in terrestrial environment $[5,19]$.

Due to lesser hardware complexity, low power consumption and achievement of higher diversity gain, hybrid combining technique has been of great interest in recent days, most especially in wideband CDMA systems $[7,15,25]$. In this system, the statistical effects of multipath phenomenon follow different fading distributions such as Rayleigh, Weibull, Rician and Nakagami-m distributions. Many researches have been carried out using Rayleigh and Rician distributions which are good for terrestrial and satellite modeling. This paper adopts the use of Nakagami-m distribution which combines both Rayleigh and Rician distributions to model terrestrial and ionospheric environments

\section{EQUAL GAIN COMBINING TECHNIQUE}

Equal Gain Combining (EGC) is a combining technique in which, signals through different branches are combined after multiplying with equal weights irrespective of the signal amplitude. However, co-phasing of all signals is needed to avoid signal cancellation. EGC is often limited in practice to coherent modulations when the phase of the received signal is in locked with the transmitted signal. In many applications, the phase of the received signal cannot be tracked accurately, and it is, therefore, not possible to perform coherent detection. It is very easy to implement an Equal Gain Combining (EGC) by using a simple phase lock summing circuit. It is similar to MRC with an exception to the weighting circuits $[3,9,11,19,23]$. The performance of EGC is a little bit lower than MRC due to equal weight applied to all paths. Fig.1 shows the block diagram of EGC. It consists of channel impulse response $h_{1}, h_{2} \ldots . h_{l}$ which have equal gains. These are being received by the antennas, which have different delays before summing. The received signals $r_{1}(t), r_{2}(t), \ldots \ldots r_{L}(t)$ are processed by Radio Frequency (RF) chain, Matched Filter (MF). The PDF of the EGC $P_{\gamma_{t} E G C}\left(\gamma_{t}\right)$ is given by [6], [12] as

$$
\begin{aligned}
& P_{\gamma_{t} E G C}\left(\gamma_{t}\right)=\frac{1}{\gamma_{a v}} \exp \left(-\frac{2 \gamma_{t}}{\gamma_{a v}}\right)+ \\
& \sqrt{\pi} \exp \left(-\frac{\gamma_{t}}{\gamma_{a v}}\right)\left(\frac{1}{2 \sqrt{\gamma_{a v} \cdot \gamma_{t}}}-\frac{1}{\gamma_{a v}} \sqrt{\frac{\gamma_{t}}{\gamma_{a v}}}\right)
\end{aligned}
$$

where:

$\gamma_{a v}$ is the average SNR at each diversity element $\gamma_{t}$ is the SNR ratio at the output of the EGC. 


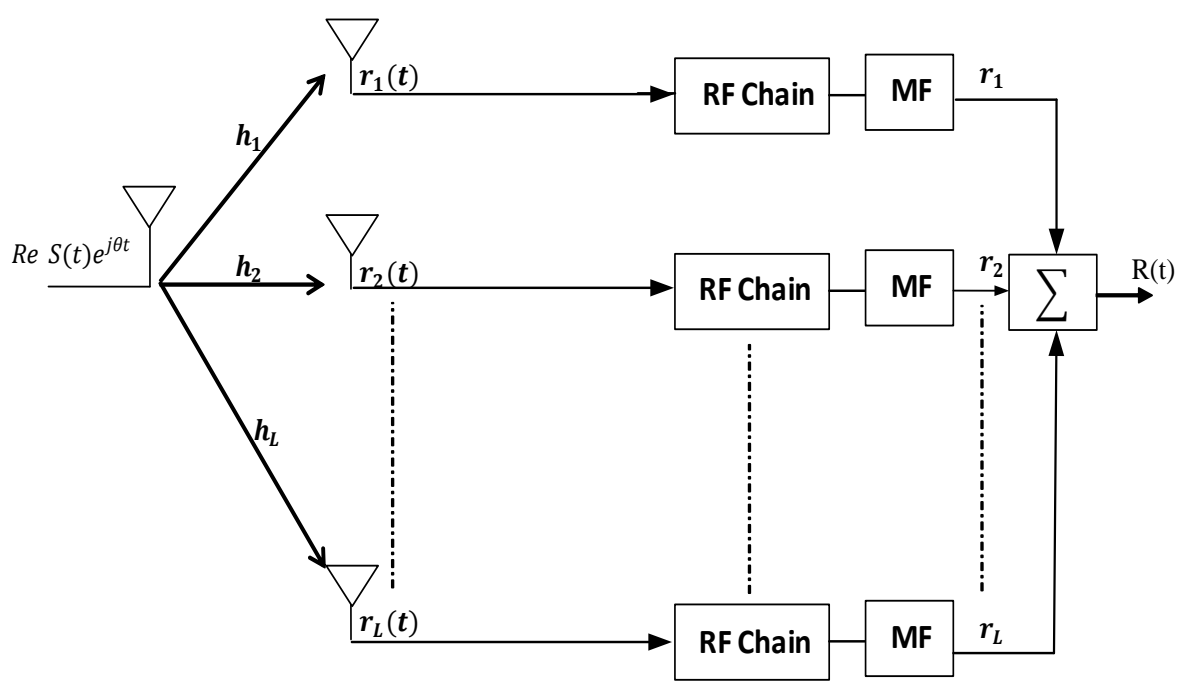

Fig.1. Block diagram of Equal Gain Combining [2]

\section{THRESHOLD COMBINING}

Threshold Combining (TC) is a technique that avoids the need for a dedicated receiver on each branch. It scans each of the branches in sequential order and gives out the signal higher than the threshold. Co-phasing is not required since only one branch output is used at a time. Once a branch is chosen, as long as the SNR on that branch remains above the desired threshold, the combiner gives out the signal. If the SNR on the selected branch falls below the threshold, the combiner switches to another branch. Selection Combining (SC) for system that transmits continuously required a dedicated receiver on each branch to continuously monitor the branch SNR $[11,16]$. SC in its conventional is impractical for systems that use uninterrupted transmission, such as FrequencyDivision Multiple-Access systems since it requires the simultaneous and continuous monitoring of all the diversity branches. Hence, SC is often implemented in the form of switching or scanning diversity, in which, rather than continually picking the best branch, the receiver selects a particular branch until its SNR drops below a predetermined threshold. When this happens, the receiver switches to another branch $[14,21,22]$. The output SNR of the combiner ' $\gamma$ ' is given by [16] as

$$
\gamma=\left\{\gamma_{1} \ldots \ldots \gamma_{l}>T\right\}
$$

where: $\gamma_{l}$ is the received SNR per bit of the $l^{\text {th }}$ branch at any instant and $\mathrm{T}$ is switching threshold.

\section{NAKAGAMI-M DISTRIBUTION}

Nakagami - $m$ distribution is a distribution that combines the attributes of Rayleigh and Rician distribution. It is also related to the gamma distribution, though, Rayleigh and Rician distributions are commonly used to describe the mobile communication channel, the Rayleigh distribution falls short in describing long- distance fading effects with sufficient accuracy [1, 6, 20]. Nakagami-m distribution provides a better accuracy to less and more severe conditions than the Rayleigh and Rician model. This is a central Chi-square distribution and the PDF of the distribution ' $P_{r}(r)$ ' is given by [17] as

$$
P_{r}(r)=\frac{2}{\Gamma(\mathrm{m})}\left(\frac{m}{2 \sigma^{2}}\right)^{m} r^{2 m-1} \exp \left(\frac{-m r^{2}}{2 \sigma^{2}}\right) r \geq 0
$$

where: $\Gamma($.$) represents the Gamma function$

$\sigma^{2}$ is the time-averaged power of the received signal $r$ is the amplitude of the received signal

$\mathrm{m}$ is the shape factor $(\mathrm{m}>1)$ which is the inverse of normalized variance of $\mathrm{r}^{2}$, when $\mathrm{m}=1$, Nakagami-m distribution degenerates to Rayleigh distribution. This distribution often gives the best fit to Land-mobile and indoor mobile multipath propagation.

\section{QUADRATURE AmPLitude MOdULATION}

Quadrature Amplitude Modulation (QAM) is one of digital modulation technique that changes the phase and amplitude of the transmitted signal simultaneously. QAM offers increased data throughput and spectral efficiency. It is accomplished by varying the amplitude of two sinusoidal waveforms that are in quadrature to each other and allows the effective transmission of two signals at the same frequency thereby doubling the rate at which the data is transmitted [8].

\section{RELATED WORK}

In [27], Performance Analysis of Diversity Techniques in wireless Communication is carried out to address the signal fluctuation in wireless communication using Threshold Combining (TC). In this paper, receivers scan each of the branches in sequential order and output the signal with SNR above a given threshold. Once a branch is chosen, the combiner gives the output as long as the 
SNR on that branch remains above the desired threshold. If the SNR on the selected branch falls below the threshold, the combiner switches to another branch. As in $\mathrm{SC}$, since only one branch output is used at a time, cophasing is not required. The result of this paper shows that, this method has a poor performance when compared to EGC but with low complexity. Also, in [8] performance analysis of conventional diversity combining Schemes in Rayleigh fading channel is carried out to solve problem of signal fluctuation at the receiver using Equal Gain Combining (EGC). In this paper, signals from different diversity branches are co-phased and multiplied with equal gain before summing. The result of this paper shows that, this method has a better performance when compared with Threshold Combining (TC) but suffered from higher complexity.

However, past researches show that, EGC has better performances than $\mathrm{TC}$ but at expense of hardware complexity. Though, TC shows a poor performance compared to EGC but with reduction in hardware complexity. Therefore, this paper develops a hybrid TCEGC technique to reduce the complexity of conventional EGC and at the same time reducing the fluctuation of the received signal, thereby improving the performance of the system.

\section{METHODOLOGY}

Development of a hybridized diversity combiner is carried out using Threshold Combining (TC) and Equal
Gain Combining (EGC). The system model for the received signal is developed over Nakagami-m fading channel. Closed form expression for the developed model is derived and used to evaluate the performance of the model. Simulation of the received signal for the developed model is carried out using MATLAB 7.2 simulation software. The model is evaluated using Outage Probability (OP) and processing Time (PT) to determine the performance.

\section{A. Development of a Hybridized Diversity Combining Technique}

The faded signal through the Nakagami-m fading channel from the transmitting antenna is received by Threshold Combiner (TC). TC scans through the Nakagami-m fading channel to select the path that is greater than the set threshold of $1 \mathrm{~dB}$ and ignore the weaker branches. Fig. 2 shows the block diagram of TC while Fig. 3 shows the block diagram of the hybridized combiner. In Fig.2, there are three branches at inputs to the TC. These serve as input to three number of TC and each TC gives an output, making a total of three branches at the output of TC. The three branches obtained at the output of Threshold Combiner serve as input to Equal Gain Combiner (EGC), this is shown in Fig. 3. From Fig.3, the three branches of signal from the output of TC are co-phased to avoid cancellation of signal due to different phases and weighted by the same weight. The signals are then passed through MF to remove the unwanted signals that might present and sum up.

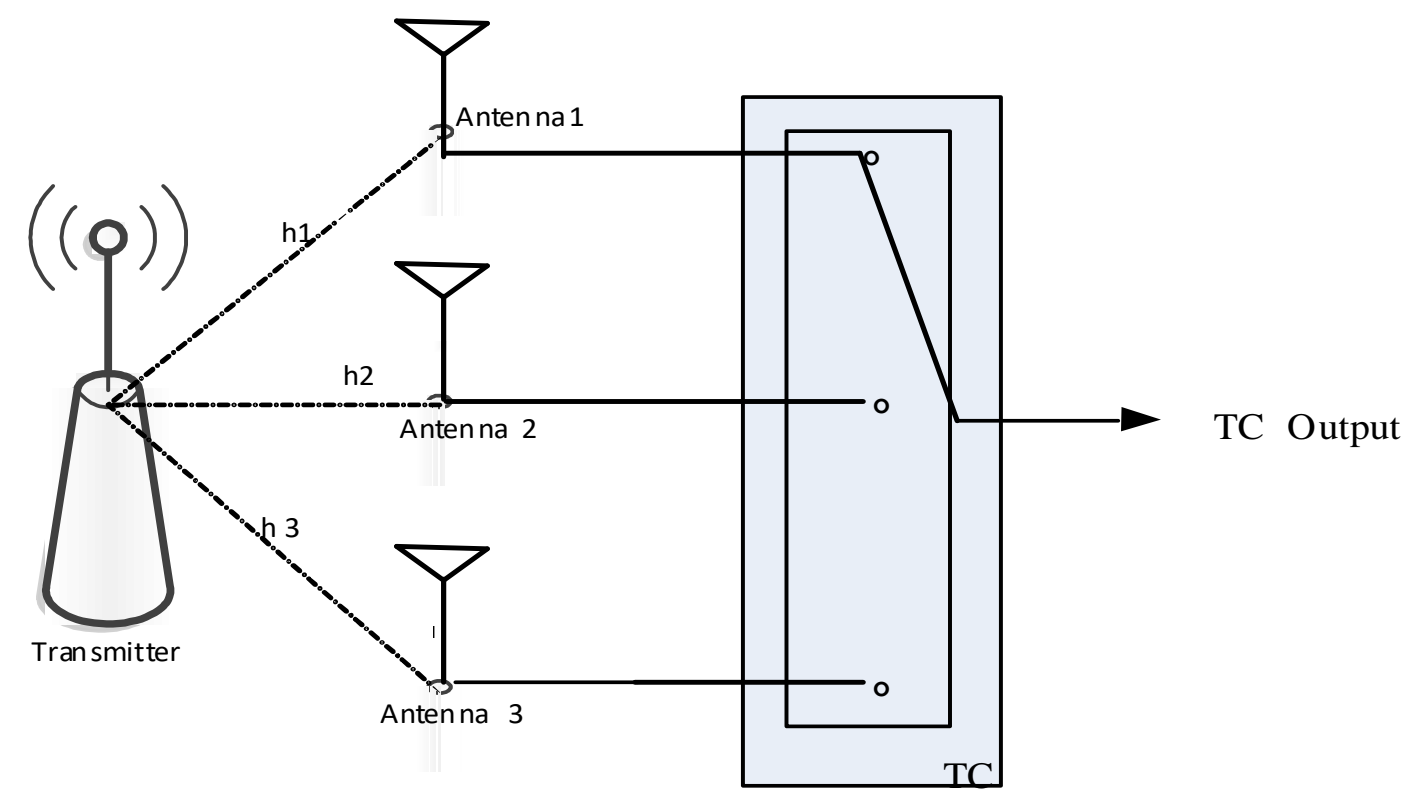

Fig.2. Threshold Combining Technique 


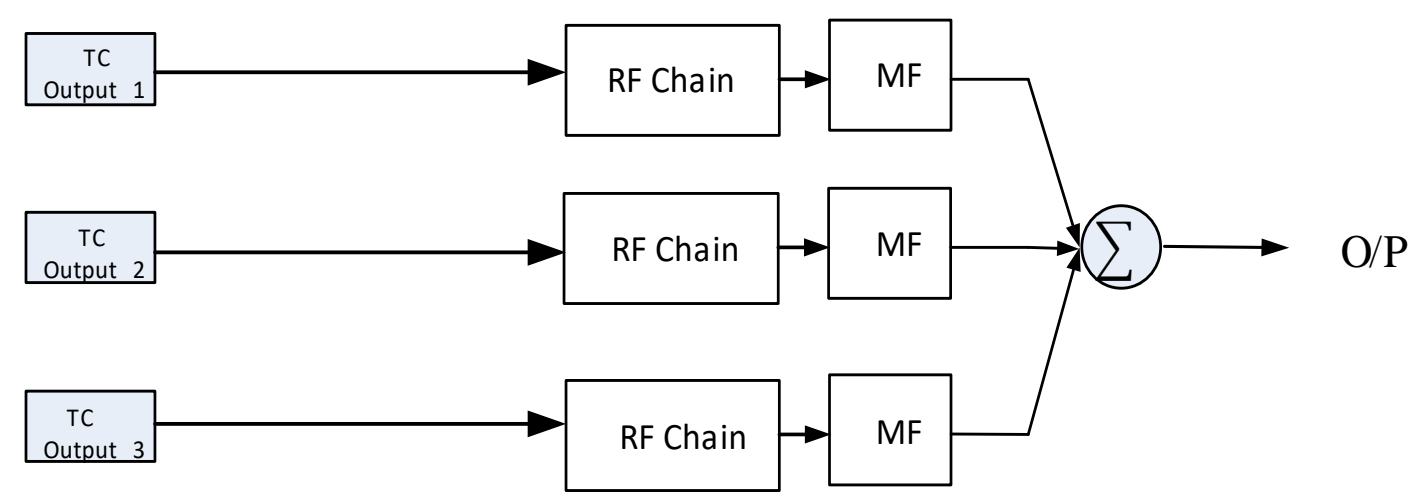

Fig.3. Hybridized Threshold-Equal Gain Combining Technique

\section{B. Probability Density Function (PDF) of the Received Signal for the Developed Model}

For hybrid TC-EGC model, signal is received by the three Threshold Combining (TC) techniques having three diversity branches. The SNR output of the TC ' $\gamma_{a v}$ ' is given by [16] and [4] as

$$
\gamma_{a v}=\left\{\gamma_{1} \ldots \ldots \gamma_{l}>T\right\}
$$

where: $\gamma_{l}$ is the received SNR per bit of the $l^{\text {th }}$ branch at any instant and ' $\mathrm{T}$ ' is switching threshold.

The inputs of EGC are the outputs of TC. Therefore, EGC with L branches, has output SNR $\gamma_{t}$ given by [5] as

$$
\gamma_{t}=\frac{\gamma^{l-1} \exp (-\gamma / \bar{\gamma})}{\bar{\gamma}^{l} \Gamma(l)}
$$

where $\gamma$ is the instantaneous SNR
$\Gamma(l)$ is the Gamma function $l$ is the diversity branch which is the output of Threshold Combining (that is, $S N R>T$ for each TC )

Probability Density Function (PDF) of the SNR output of the combiner $P_{r E G C}\left(\gamma_{t}\right)$ over nakagami-m fading channel is given by [10] as

$$
\begin{gathered}
P_{r E G C}\left(\gamma_{t}\right)=\frac{1}{\bar{\gamma}} \exp \left(-2 \gamma_{t} / \bar{\gamma}\right)+ \\
(\pi)^{\frac{1}{2}} \exp \left(-\gamma_{t} / 2 \bar{\gamma}\right)\left(\frac{1}{2\left(\bar{\gamma} \gamma_{t}\right)^{\frac{1}{2}}}-\frac{1}{\bar{\gamma}}\left(\frac{\gamma_{t}}{\bar{\gamma}}\right)^{\frac{1}{2}}\right)
\end{gathered}
$$

where: $\bar{\gamma}$ is the average SNR at each diversity branch

$\gamma_{t}$ is the SNR at the output of the EGC.

Substituting Equation 5 into Equation 6 gives

$\bar{\gamma}$ is the average SNR

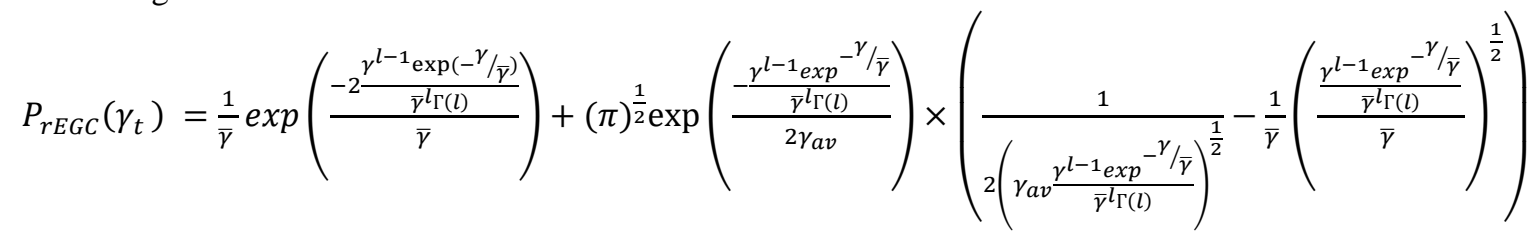

$$
\begin{aligned}
& O P=P\left(\gamma<\gamma_{0}\right)=\int_{0}^{\gamma_{0}} P_{r}(\gamma) d \gamma
\end{aligned}
$$
hybridized Threshold-Equal Gain Combining

\section{Outage Probability $(O P)$}

Outage Probability (OP) is the probability that the Signal-to-Noise Ratio (SNR) of the received signal falls below a given threshold. According to [14], outage probability ' $\mathrm{OP}$ ' is given as where: $\gamma$ is the SNR of the received signal $\gamma_{0}$ is the set threshold

$P_{r}(\gamma)$ is the PDF of the received signal

Substituting Equation (7) into Equation (8) gives

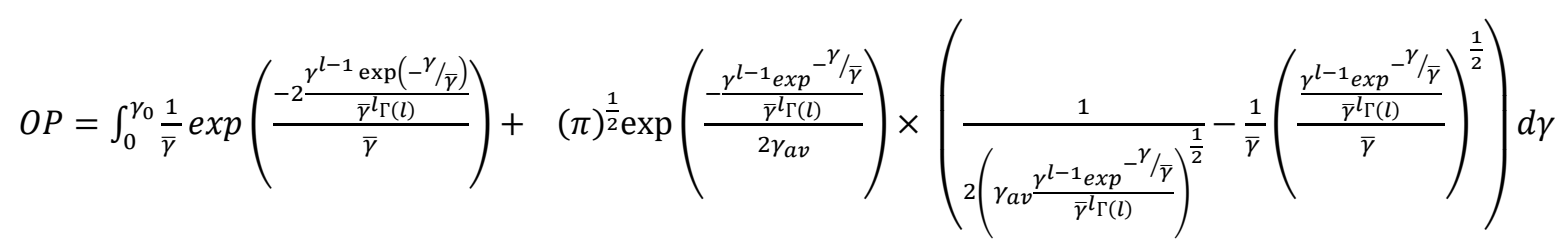


Equation (9) is the outage probability for the hybridized TC-EGC

\section{RESULTS AND DISCUSSION}

\section{A. Outage Probability Results for Two Propagation} Paths Using M-QAM Schemes

Fig.4 presents the Outage Probability (OP) versus SNR for TC, EGC and Hybridized TC-EGC technique with $L=2$ using 4QAM and 16QAM schemes over Nakagami-m fading channel. At SNR of $4 \mathrm{~dB}$ using 4QAM signaling scheme, the OP values of 0.6316 , 0.6152 and 0.5801 are obtained for TC, EGC and TCEGC, respectively, while at 16QAM scheme, OP values of $0.6313,0.6021$ and 0.5761 are obtained for TC, EGC and TC-EGC, respectively. The results obtained show that, TC-EGC gives lower OP values compared to TC and EGC. The performance of TC-EGC is due to combining nature of the technique that increases the signal strength. The results obtained are contained in Table 1.

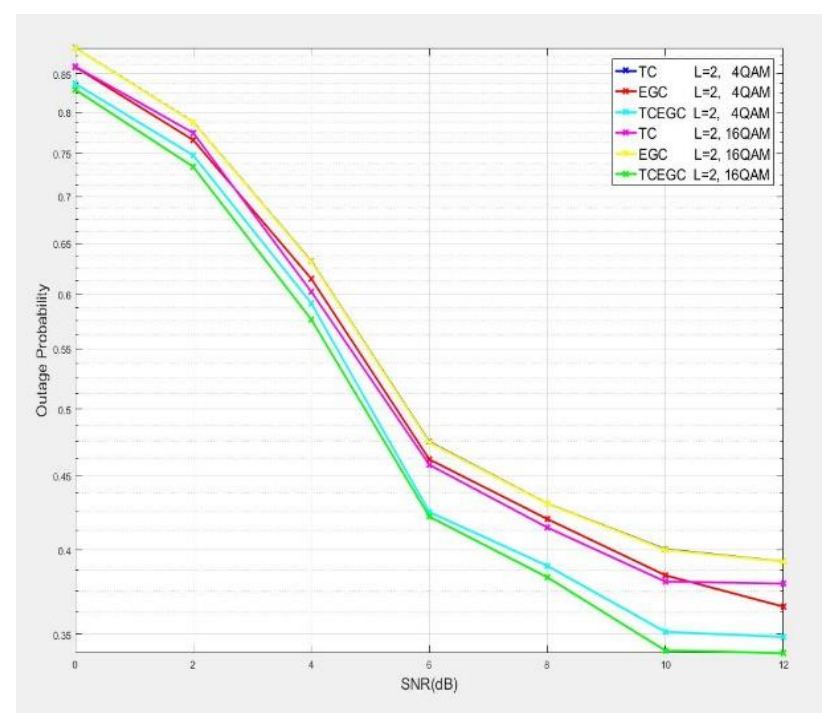

Fig.4. Outage Probability versus SNR for TC, EGC and hybridized TCEGC at $L=2$ using 4, 16 QAM signaling Schemes over Nakagami-m Fading Channel.

Table 1. Outage Probability for TC, EGC, Hybrid TC- EGC at L of 2 with 4QAM, 8QAM and 16QAM Signaling schemes

\begin{tabular}{|c|c|c|c|c|c|c|c|c|c|}
\hline & \multicolumn{3}{|c|}{$\mathrm{L}=2.8 \mathrm{QAM}$} & \multicolumn{3}{|c|}{$\mathrm{L}=2.4 \mathrm{QAM}$} & \multicolumn{3}{|c|}{$\mathrm{L}=2.16 \mathrm{QAM}$} \\
\hline & EGC & TC & TC-EGC & EGC & $\mathrm{TC}$ & TC-EGC & EGC & $\mathrm{TC}$ & TC-EGC \\
\hline 0 & 0.8594 & 0.8856 & 0.8369 & 0.8755 & 0.8856 & 0.8316 & 0.8601 & 0.8854 & 0.8291 \\
\hline 2 & 0.7655 & 0.7873 & 0.7472 & 0.7735 & 0.7872 & 0.7405 & 0.7741 & 0.7871 & 0.7337 \\
\hline 4 & 0.6146 & 0.6317 & 0.5911 & 0.6152 & 0.6316 & 0.5801 & 0.6021 & 0.6313 & 0.5761 \\
\hline 6 & 0.4617 & 0.4747 & 0.4248 & 0.4636 & 0.4746 & 0.4289 & 0.4578 & 0.4743 & 0.4217 \\
\hline 8 & 0.4201 & 0.4304 & 0.3901 & 0.4058 & 0.4304 & 0.3852 & 0.4145 & 0.4303 & 0.3831 \\
\hline 10 & 0.3843 & 0.4004 & 0.3514 & 0.3875 & 0.4003 & 0.3551 & 0.3805 & 0.4001 & 0.3411 \\
\hline 12 & 0.3657 & 0.3927 & 0.3486 & 0.3731 & 0.3927 & 0.3391 & 0.3793 & 0.3926 & 0.3398 \\
\hline
\end{tabular}

B. Outage Probability Results for Three Propagation Paths Using M-QAM Signaling schemes

Fig.5 depicts the OP versus SNR for TC, EGC and Hybridized TC-EGC with three propagation paths ' $L$ ' $=3$ using 4QAM and 16QAM schemes over Nakagami-m fading channel. The OP values obtained with 4QAM signaling scheme at SNR of $4 \mathrm{~dB}$ are $0.5135,0.4862$ and
0.4658 for TC, EGC and TC-EGC, respectively, while at 16QAM, OP values of $0.5058,0.4906$ and 0.4538 are obtained for TC, EGC and TC-EGC, respectively. The results obtained show that $\mathrm{OP}$ values decrease as number of path increases. The results obtained are also presented in Table 2.

Table 2. Outage Probability for TC, EGC, Hybrid TC- EGC at L of 3 with 4QAM, 8QAM and 16QAM Signaling schemes

\begin{tabular}{|c|c|c|c|c|c|c|c|c|c|}
\hline & \multicolumn{3}{|c|}{$\mathrm{L}=3.4 \mathrm{QAM}$} & \multicolumn{3}{|c|}{$\mathrm{L}=3.8 \mathrm{QAM}$} & \multicolumn{3}{|c|}{$\mathrm{L}=3.16 \mathrm{QAM}$} \\
\hline & EGC & $\mathrm{TC}$ & TC-EGC & EGC & $\mathrm{TC}$ & TC-EGC & EGC & $\mathrm{TC}$ & TC-EGC \\
\hline 0 & 0.6543 & 0.6781 & 0.6303 & 0.6621 & 0.6796 & 0.6384 & 0.6536 & 0.6701 & 0.6164 \\
\hline 2 & 0.5008 & 0.5149 & 0.4663 & 0.4988 & 0.515 & 0.4649 & 0.4935 & 0.5145 & 0.4656 \\
\hline 4 & 0.4862 & 0.5135 & 0.4658 & 0.4899 & 0.5149 & 0.4595 & 0.4906 & 0.5058 & 0.4538 \\
\hline 6 & 0.3971 & 0.425 & 0.3835 & 0.4138 & 0.4259 & 0.374 & 0.3981 & 0.4201 & 0.3733 \\
\hline 8 & 0.3848 & 0.4008 & 0.3524 & 0.373 & 0.4011 & 0.3414 & 0.3809 & 0.3992 & 0.3434 \\
\hline 10 & 0.3597 & 0.3729 & 0.3156 & 0.3576 & 0.3731 & 0.3241 & 0.3614 & 0.3718 & 0.3293 \\
\hline 12 & 0.3247 & 0.337 & 0.2816 & 0.3149 & 0.3376 & 0.2813 & 0.3195 & 0.3333 & 0.2843 \\
\hline
\end{tabular}




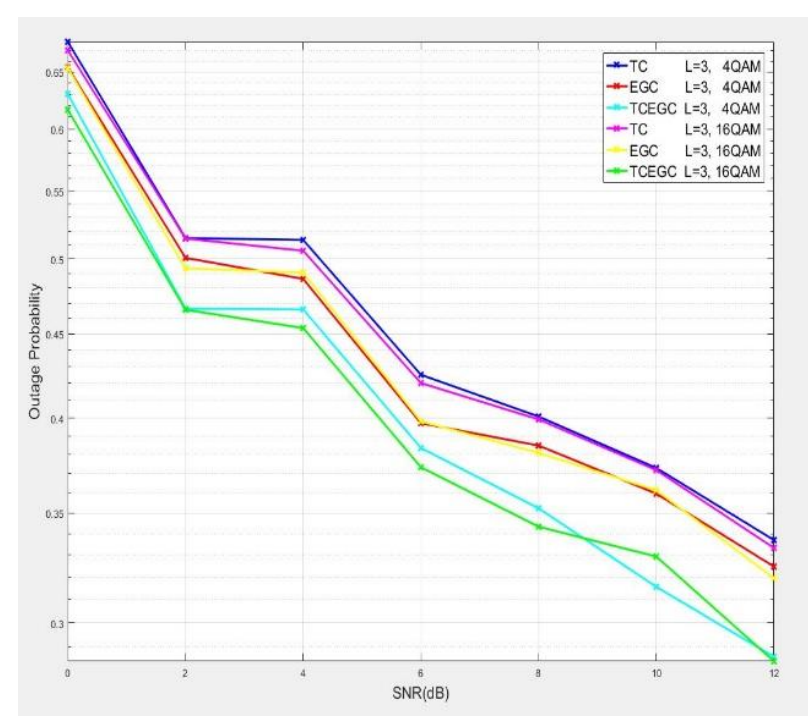

Fig.5. Outage Probability versus SNR for TC, EGC and hybridized TCEGC at $L=3$ using 4, 16 QAM signaling Schemes over Nakagami-m Fading Channel.

\section{Outage Probability Results for Four Propagation Paths Using M-QAM Signaling}

Fig. 6 shows the Outage Probability (OP) versus SNR for TC, EGC and Hybridized TC-EGC with L of 4 using 4QAM, 16QAM signaling schemes over Nakagami-m fading channel. At SNR of $4 \mathrm{~dB}$, the OP values of 0.4709 0.4494 and 0.4151 are obtained, for TC, EGC and TCEGC, respectively, while at 16QAM scheme, the OP values of $0.4719,0.4518$ and 0.4216 are obtained for TC, EGC and TC-EGC, respectively. Table 3 also contains the same results.

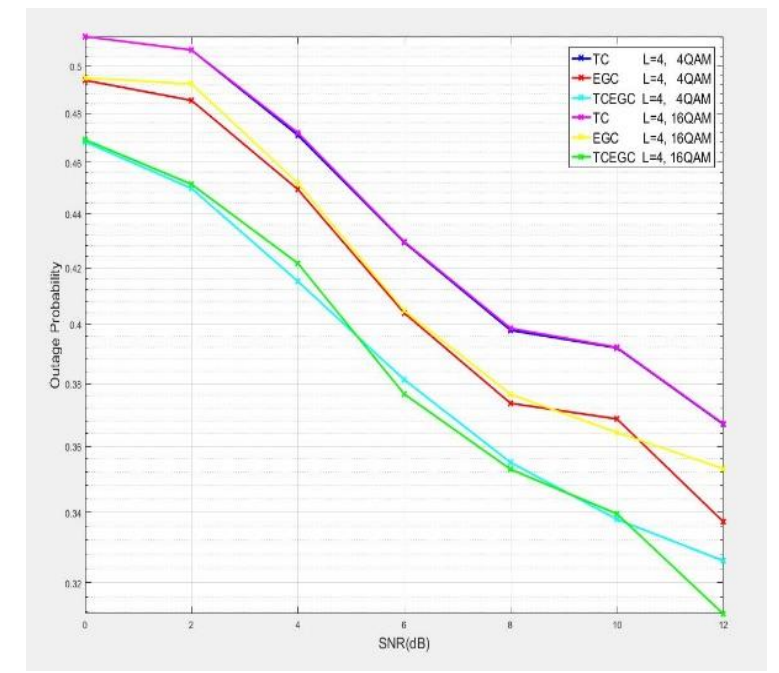

Fig.6. Outage Probability versus SNR for TC, EGC and hybridized TCEGC at $L=4$ using 4, 16 QAM signaling Schemes over Nakagami-m Fading Channel.

Table 3. Outage Probability for TC, EGC, Hybrid TC- EGC at L of 4 with 4QAM, 8QAM and 16QAM Signaling scheme

\begin{tabular}{|c|c|c|c|c|c|c|c|c|c|}
\hline & \multicolumn{3}{|c|}{$\mathrm{L}=4.4 \mathrm{QAM}$} & \multicolumn{3}{|c|}{$\mathrm{L}=4.8 \mathrm{QAM}$} & \multicolumn{3}{|c|}{$\mathrm{L}=4.16 \mathrm{QAM}$} \\
\hline & EGC & TC & TC-EGC & EGC & TC & TC-EGC & EGC & $\mathrm{TC}$ & TC-EGC \\
\hline 0 & 0.4938 & 0.5128 & 0.4682 & 0.4935 & 0.5129 & 0.4573 & 0.4948 & 0.5128 & 0.4691 \\
\hline 2 & 0.4853 & 0.5069 & 0.4498 & 0.4793 & 0.5069 & 0.4502 & 0.4923 & 0.5069 & 0.4514 \\
\hline 4 & 0.4494 & 0.4709 & 0.4151 & 0.4599 & 0.4729 & 0.4215 & 0.4518 & 0.4719 & 0.4216 \\
\hline 6 & 0.4039 & 0.4293 & 0.3813 & 0.4048 & 0.4299 & 0.3829 & 0.4047 & 0.4296 & 0.3766 \\
\hline 8 & 0.3736 & 0.3979 & 0.355 & 0.3721 & 0.3993 & 0.3412 & 0.3765 & 0.3986 & 0.3529 \\
\hline 10 & 0.3686 & 0.3919 & 0.338 & 0.3664 & 0.3923 & 0.3407 & 0.3643 & 0.3921 & 0.3396 \\
\hline 12 & 0.3373 & 0.3669 & 0.3261 & 0.3428 & 0.3673 & 0.3113 & 0.3531 & 0.3671 & 0.3115 \\
\hline
\end{tabular}

\section{Processing Time for different Propagation Paths using 4QAM Signaling}

The Processing Time (PT) versus SNR for EGC and hybridized TC-EGC are presented using bar chart in Figs. 7-9 at different paths for 4QAM modulation scheme. Fig. 7 presents the PT with L of 2 using 4QAM scheme over Nakagami-m fading channel for conventional EGC and hybridized TC-EGC. At SNR of $4 \mathrm{~dB}$, the PT values obtained are $0.8956 \mathrm{~s}$ and $0.7894 \mathrm{~s}$ for EGC and TC-EGC, respectively. Fig. 8 shows the PT with L of 3 using 4QAM scheme over Nakagami-m fading channel for conventional EGC and hybridized TC-EGC. At SNR of 4 $\mathrm{dB}, \mathrm{PT}$ values of $0.8105 \mathrm{~s}$ and $0.6900 \mathrm{~s}$ are obtained for EGC and TC-EGC, respectively. Fig. 9 depicts the processing time with $\mathrm{L}$ of 4 with 4QAM scheme over Nakagami-m fading channel for conventional EGC and hybridized TC-EGC. At SNR of $4 \mathrm{~dB}$, the PT values obtained are $0.6745 \mathrm{~s}$ and $0.5692 \mathrm{~s}$ for EGC and TC-EGC,

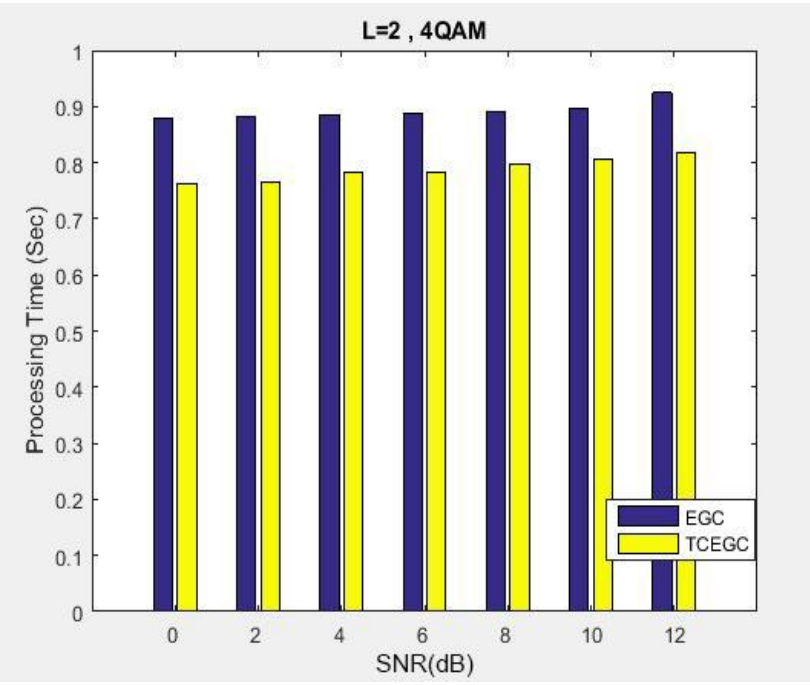

Fig.7. Processing time versus SNR for $L=2$ using 4QAM Signaling scheme over Nakagami-m fading channel. 
respectively. The result is justifiable in that; the hardware complexity for the conventional EGC has been enhanced using the hybridized technique where TC first selects the path with strongest SNR value. The enhancement of EGC is shown from the lower processing time values which indicate better performance.

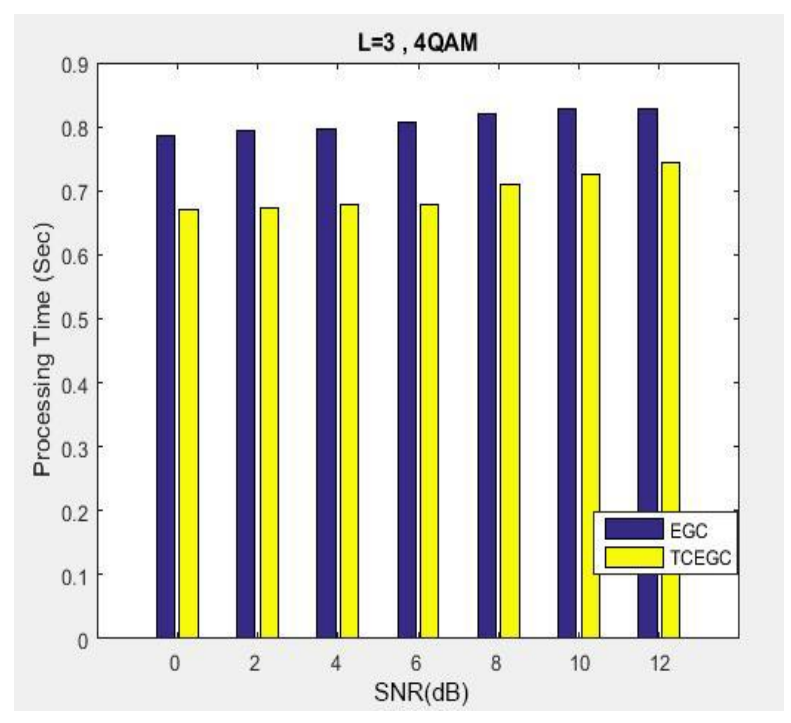

Fig.8. Processing time versus SNR for $L=3$ using 4QAM signaling scheme over Nakagami-m fading channel.

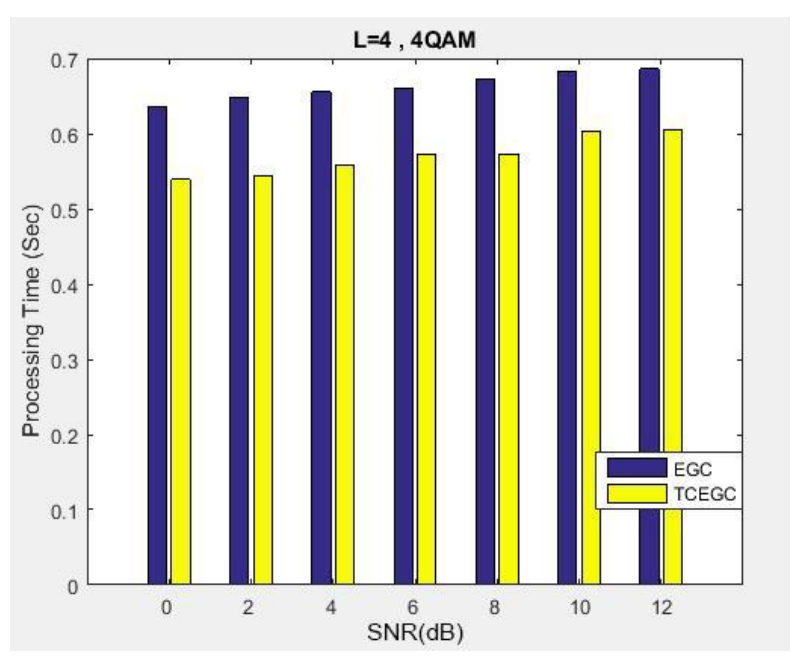

Fig.9. Processing time versus SNR for $L=4$ using 4QAM signaling scheme over Nakagami-m fading channel.

\section{CONCLUSIONS}

A hybridized TC-EGC technique has been developed using Threshold Combining (TC) and Equal Gain Combining (EGC) over Nakagami-m fading channel. A closed form expression for Probability Density Function (PDF) of the received signal is derived for the hybridized model and this is used to derive Outage Probability (OP) for the hybridized technique. The technique is simulated using MATLAB simulation software and evaluated using OP and PT to determine the performance. OP for TC, EGC, hybridized TC-EGC are obtained at different SNR for different paths using 4, 16 QAM signaling schemes. The results obtained show that the hybridized TC-EGC performs better than TC and EGC due to lower OP and PT values. The performance of the hybridized TC-EGC is as a result of selection of the strongest path while ignoring the weak paths. The results of this study show that, the technique gives better performance as the number of paths increases. This is in agreement with the work of [8], where analysis of conventional diversity combining scheme in Rayleigh fading channel is studied. Therefore, the hybridized TC-EGC has been shown to have a better performance.

\section{REFERENCES}

[1] S.F. Abdel-Hakim. Performance Study of Nakagami-m Fading Channels with Correlated Unbalanced Branches, PhD Thesis Submitted to Jordan University of Science and Technology: 3-20. 2003.

[2] Z. K Adeyemo, and I.A. Ojedokun., EGC Receiver using Single Radio Frequency Chain and Single Matched Filter over Combined Rayleigh and Rician Fading Channels, ARPN Jorurnal of Engineering and Applied Sciences, 9(7): 992-994. 2014.

[3] Z. K Adeyemo and T. I. Raji "Effects of Diversity combining in mobile Terrestrial Environment" Continental Journal of Engineering Sciences 4(5): 27-37. 2010.

[4] A.J. Goldsmith "Wireless Communication" first Edition, Cambridge University press, Cambridge, England: 2005, pp 205-214.

[5] P.V. Hima and P. Seema Performance Analysis of Hybrid MRC/EGC Diversity Combining Technique over AWGN channel, IOSR Journal of Electronics and Communication Engineering 42(2):25-26. 2016.

[6] A. Hussien, Performance Analysis of Energy Detection Over Different Generalized Wireless Channel Based Spectrum Sensing in Cognitive Radio, PhD thesis submitted to Department of Electronic and Computer Engineering, Brunel University, London, United Kingdom. 2015, pp 34-37.

[7] S. Mohamed, and K.S. Marvin Performance of Coherent Receiver with Hybrid SC/MRC Over Nakagami-m Fading Channel, IEEE Transactions on Vehicular Technology 48(4):1155-1157. 1999.

[8] S. Nitika and S. Deepak Performance Analysis of Conventional Diversity Combining Schemes in Rayleigh Fading Channel, International Journal of Advanced Research in Computer Science and software Engineering 2(6):197-198. 2012.

[9] M. Paul, H.A. Matti., D. Guido and L. Lukas AntennaPattern Diversity Versus Space Diversity for Use at Handhelds, IEEE Transaction on Vehicular Technology 53(4) pp 1036-1037. 2004.

[10] J. G. Proakis,. "Digital Communications", Mc Graw-Hill companies, inc., International Edition, 2001 pp 87:98.

[11] V. Ramanthan Performance Evaluation of Equal Gain Diversity Systems in Fading Channel, Master Thesis Submitted to the Faculty of the Virgiria Polytechnic, Institute and State University pp 1-4, 2003.

[12] T.S. Rappaport, "Wireless communications principle and practice, Even publishers Chicago. 2002, pp 240-250.

[13] S. Rupaban, Performance Analysis of Diversity Combining Receiver over Hoyt, $\delta-\mu$ and $k-\mu$ Fading Channels, PhD thesis submitted to the Department of Technology, Guwahati, India, 2011, pp 45-68. 
[14] M.K. Simon, and M.S. Alouini, "Digital Communication over fading channels second edition "John Wiley \& Sons. Inc. Hoboken, New Jersey, 2004, pp 313-317.

[15] P.J. Suvarna and S.H. Vaibhav, Performance of Maximum Ratio Combining (MRC) MIMO Systems for Rayleigh Fading Channel, International Journal of Scientific and Research Publications, 3(2):1-3. 2013.

[16] G. L. Stuber, "Principles of Mobile Communications, "Second Edition, Kluwer Academic Publishers New York: 2002, pp 245-267.

[17] T. Taruna, and P. Bhumika., Multiple Detectors Based Analytical Performance of Spectrum Sensing, International Journal of Advanced Computer Research 4 (1), 95-98. 2014.

[18] T. Pratt, C. W. Boastian, and J.E. Allnutt., "Satellite Communications, "Second Edition, John Wiley \& Sons, New Jersey. 2003, PP 34-67

[19] J. Islam, Performance Analysis of Diversity Technique for Wireless Communication System, Master Thesis Submitted to the Department of Technology Engineering, Blackline Institute of Technology. 2009, PP 50-54.

[20] A. Ibrahim, Performance of M-QAM Over Generalized Mobile Fading Channel Using MRC Diversity', Master thesis Submitted to the department of Electrical Engineering, College of Engineering, King Saud University. 2007, PP 7-12.

[21] A. Annamalai and C Tellambura, "Performance evaluation of generalized selection Diversity Systems over Nakagami m fading channels", Journal of Wireless Communications and Mobile Computing, 3(2): 99 - 116. 2003.

[22] A. Annamalai, Gantam Deora and C. Tallambura, Analysis of Generalized Selection Diversity Systems in Wireless Channel, IEEE Transactions on Vehicular Technology, 55(6):1765-1766. 2006.

[23] M. Shukla, G. Akanksha and B. Rinkoo, Performance Evaluation of Maximal Ratio Receiver Combining Diversity with Prime Interleaver for Iterative IDMA Receiver, Journal of Information Engineering and Application 1(3):29-33. 2011.

[24] L.J. Ippolito,., "Satellite communication system engineering: Atmospheric Effect, Satellite link Design, and System Performance," first edition John Wiley \& Sons, Singapore: 2008, PP 103-120.

[25] G. Rajkumar, Performance Evaluation of Maximum Ratio Combining Scheme in WCDMA System for different Modulation, International Journal of Emerging Technologies in Engineering Research 4(5), 2016, PP 240-242.

[26] K. Pavan, and T.R. thammi, FPGA Implementation of SRRC Filter for WCDMA Systems, International Journal of Science and Research 4 (3): 2582-2584. 2013.

[27] Jaherul, I. Performance Analysis of Diversity Techniques for Wireless Communication System, Journal of Wireless Communications and Mobile Computing, 12(4), 2012, pp 1-6.

\section{Authors' Profiles}

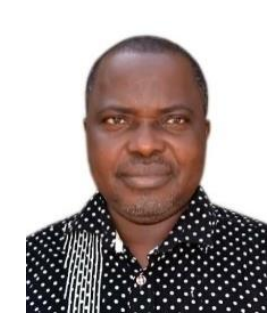

Zachaeus K. Adeyemo received the B.Eng. and M.Eng. degrees in Electrical Engineering from University of Ilorin, Ilorin Nigeria and his Ph.D. degree in Electronic and Electrical Engineering in 2009 from Ladoke Akintola University of Technology (LAUTECH), Ogbomoso, Nigeria. He is a member of the IEEE and registered member of Council for the Regulation of Engineering in Nigeria (COREN). Dr. Adeyemo is an associate Professor of Electronic and Electrical Engineering. His research interest is on signal processing in wireless communications.

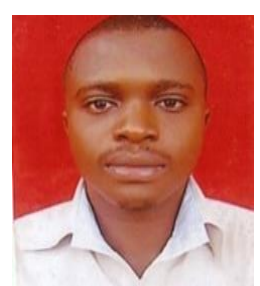

Samson I. Ojo received his B.Tech and M.Tech degrees in Electronic and Electrical Engineering in 2011 and 2018, respectively, from Ladoke Akintola University of Technology (LAUTECH), Ogbomoso, Nigeria. He is a registered member of Council for the Regulation of Engineering in Nigeria (COREN). He intends pursuing his Ph.D in signal processing in mobile communication.

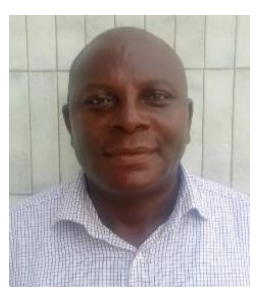

Simeon B. Ebinaiye received his post graduate Diploma (PGD) in Electronic and Electrical Engineering from Ladoke Akintola University of Technology (LAUTECH). Currently he is pursuing his Postgraduate Degree programme in wireless communication from the same University.

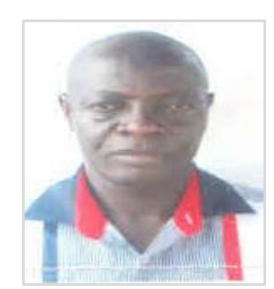

Olasunkanmi F. Oseni received the B.Eng. and M.Eng. degrees in Electrical Engineering from University of Ilorin, Ilorin Nigeria and his Ph.D. degree in Electronic and Electrical Engineering in 2018 from Ladoke Akintola University of Technology (LAUTECH), Ogbomoso, Nigeria. He is a member of the IEEE and registered member of Council for the Regulation of Engineering in Nigeria (COREN).

How to cite this paper: Zachaeus K. Adeyemo, Samson I. Ojo, Simeon B. Ebinaiye, Olasunkanmi F. Oseni, "Development of a Hybridized Diversity Combiner over Nakagami Fading Channel", International Journal of Information Engineering and Electronic Business(IJIEEB), Vol.11, No.3, pp. 45-53, $2019 . \quad$ DOI: 10.5815/ijieeb.2019.03.06 